\title{
B-scan-sectioned dynamic micro-optical coherence tomography for bulk-motion suppression
}

\author{
Chaoliang Chen (陈朝良) $)^{1^{*}}$, Weisong Shi (史伟松) $)^{2}$, Zhiyuan Qiu (仇志远) $)^{2}$, Victor X. D. Yang (杨晓东) $)^{3,4,5}$, and \\ Wanrong Gao (高万荣) ${ }^{2}$ \\ ${ }^{1}$ Advanced Photonics Center, School of Electronic Science \& Engineering, Southeast University, Nanjing 210096, China \\ ${ }^{2}$ Department of Optical Engineering, Nanjing University of Science and Technology, Nanjing 210094, China \\ 3 Department of Electrical, Computer, and Biomedical Engineering, Ryerson University, Toronto, Ontario M5B 2K3, Canada \\ ${ }^{4}$ Division of Neurosurgery, Sunnybrook Health Sciences Centre, Toronto, Ontario M4N 3M5, Canada \\ ${ }^{5}$ Division of Neurosurgery, Faculty of Medicine, University of Toronto, Toronto, Ontario M5T 1P5, Canada \\ *Corresponding author: chaoliangchen@seu.edu.cn \\ Received October 8, 2021 | Accepted December 2, 2021 | Posted Online December 17, 2021
}

\begin{abstract}
In this Letter, we present B-scan-sectioned dynamic micro-optical coherence tomography (BD-MOCT) for high-quality subcellular dynamic contrast imaging. Dynamic micro-optical coherence tomography (D-MOCT) is a functional optical coherence tomography (OCT) technique performed on high-resolution (micron level] OCT systems; hundreds of consecutive B-scans need to be acquired for dynamic signal extraction, which requires relatively long data acquisition time. Bulk motions occurring during data acquisition (even at the micron level) may degrade the quality of the obtained dynamic contrast images. In BD-MOCT, each full B-scan is divided into several sub-B-scans, and each sub-B-scan repeats multiple times before the sample beam moves to the next sub-B-scan. After all of the sub-B-scans for a full B-scan are completely acquired, we stitch all of the sub-B-scans into the same number of full B-scans. In this way, the time interval between two consecutive stitched B-scans could be reduced multiple times for bulk-motion suppression. The performed scanning protocol modulates the scanning sequences of fast scanning and repeat scanning for improving the dynamic contrast image quality, while the total data acquisition time remains almost the same.
\end{abstract}

Keywords: optical coherence tomography; high-resolution imaging; dynamic imaging; bulk-motion suppression. DOI: 10.3788/COL202220.021102

\section{Introduction}

Dynamic optical coherence tomography $(\mathrm{D}-\mathrm{OCT})^{[1]}$ is a recently proposed functional optical coherence tomography (OCT) technique, in which multiple B-scans from the same location are acquired for calculating the power spectrum (commonly with fast Fourier transform, FFT) to contrast the sub-cellular dynamic information. This imaging modality was first validated on full-field OCT (FFOCT $)^{[1]}$. D-OCT images have the advantages of being speckle-free and high contrast compared to OCT structural images. At present, dynamic FFOCT has already been successfully applied in multiple applications ${ }^{[2-4]}$.

Micro-optical coherence tomography (MOCT) proposed by Liu et al. ${ }^{[5]}$ is a spectral domain OCT (SDOCT) with micronlevel resolutions. Compared to FFOCT, MOCT has a higher scanning speed and wider imaging field. Therefore, MOCT is also a potential technique for sub-cellular dynamic imaging. In 2020, two groups simultaneously demonstrated the applications of the super-continuum light-source-based MOCT for sub-cellular dynamic imaging ${ }^{[6,7]}$, termed D-MOCT. In Münter's work ${ }^{[6]}, 150$ consecutive B-scans were acquired for calculating the power spectrum at each spatial location, and the reflection of the glass slide was used for bulk-motion compensation in the axial direction. In Leung's work ${ }^{[7]}, 1000$ B-scans were acquired for the same calculation, and short-time Fourier transform was performed for suppressing motion artefacts.

Similar to the moving scatterers, bulk motions can also modulate the backscattered intensities, resulting in degradation on the image quality of the dynamic contrast images. The influence of bulk motions on D-MOCT imaging is inevitable and severe due to the following: (1) data acquisition time is relatively long for $\mathrm{D}$ MOCT, because hundreds of B-scans from each position need to be acquired; (2) the spatial resolution of the D-MOCT system is at the micron level, so even slight bulk shifts could effectively blur the obtained images, such as tissue elasticity and environmental vibrations (especially from the systems on mobile carts in clinics). Furthermore, it is also challenging to precisely 
compensate bulk motions with image registration algorithms because sub-pixel location shifts occur.

In this Letter, we present a B-scan-sectioned D-MOCT (BDMOCT) for achieving high-quality dynamic contrast images. In the proposed technique, a B-scan-sectioned scanning protocol was performed, where each full B-scan was evenly divided into several sections (termed sub-B-scans), and each sub-B-scan was repeated hundreds of times before the sample beam moved to the next sub-B-scan. After all sub-B-scans were completely acquired, we stitched all of the sub-B-scans into the same number of full B-scans for the following data process. In this way, the time interval between two consecutive stitched B-scans could be reduced multiple times compared to the conventional raster scanning protocol, achieving bulk-motion suppression and image quality improvement. Meanwhile, the total data acquisition time stayed almost the same.

\section{Methods}

Figure 1(a) illustrates the system structure we used in this study. The optical beam with a spectral bandwidth of 400-2300 nm from a super-continuum light source (SC400-2, Fianuim, UK) hits a dichroic mirror (DMLP1000T, Thorlabs, US), and the spectrum below $1 \mu \mathrm{m}$ is reflected and coupled by $\mathrm{L} 1$ with a focal length of $10 \mathrm{~mm}$ into one arm of a $2 \times 2$ fiber coupler (split ratio: 90:10). Ten percent of the optical power is collimated by L2 (focal length: $19 \mathrm{~mm}$ ) and then goes into a Michelson interferometer, where the beam is split into reference and sample beams by a 50:50 beam splitter cube. Two objective lenses (NA $\sim 0.31$ ) focus the two beams on a mirror and the sample, respectively, and the achieved lateral resolution is $\sim 1 \mu \mathrm{m}$. In the sample arm, the beam is reflected by a galvo-mirror (Thorlabs, US) for transverse scanning. The reflected light from the two arms interferes at the cube splitter and goes back to the fiber coupler.
A home-built spectrometer (spectral range: $725-1000 \mathrm{~nm}$ ) is used for fringe detection, which achieves an axial resolution of $\sim 1.7 \mu \mathrm{m}$ with an approximated refractive index of 1.4 for the tissue sample. The camera (EM4, e2v, UK) has 4096 pixels and runs at $37 \mathrm{kHz}$. The grating (1002-1, Wasatch, US) has a resolution of 1200 lines $/ \mathrm{mm}$. In the sample arm, the optical power on the sample is $\sim 5 \mathrm{~mW}$, and the achieved sensitivity is $\sim 91 \mathrm{~dB}$, measured by inserting an optical neutral-density filter with $\mathrm{OD}=2$ in the sample arm.

Figure 1(b) shows the B-scan-sectioned scanning protocol used in BD-MOCT. Each full B-scan is divided into $M$ sub-Bscans, and each sub-B-scan is repeated $N$ times before the sample beam moves to the next sub-B-scan. After all sub-B-scans are completely acquired, we stitch all of the sub-B-scans into the same number of full B-scans. Compared to conventional raster scanning, the time interval between the consecutive stitched Bscans can be reduced about $M$ times. Consequently, the bulk motions can be effectively suppressed to improve the quality of dynamic contrast images. In this study, each full B-scan was uniformly divided into $M=8$ sub-B-scans, and each subB-scan was repeated $N=200$ times for dynamic information extraction. To achieve a perfect mosaic of multiple sub-B-scan images, the galvo-driving voltages of the two adjacent sub-Bscans were slightly overlapped, and four extra A-scans on each side of each sub-B-scan were acquired for compensating the blurred parts caused by the inertia of the galvo-mirror. Therefore, the raw A-scan number of each full B-scan was 1088, and the A-scan number for the stitched B-scan was 1024. The conventional scanning protocol with the A-scan number of 1024 was also performed on the same tissue sample, and the obtained images were compared for demonstrating the performance of the proposed technique. The duty cycle of the sawtooth wave (galvo-driving signal) for both scanning protocols was $77 \%$, and the resultant time intervals between two conventional B-scans and two stitched B-scans were $\sim 26.6 \mathrm{~ms}$ and $\sim 3.5 \mathrm{~ms}$,

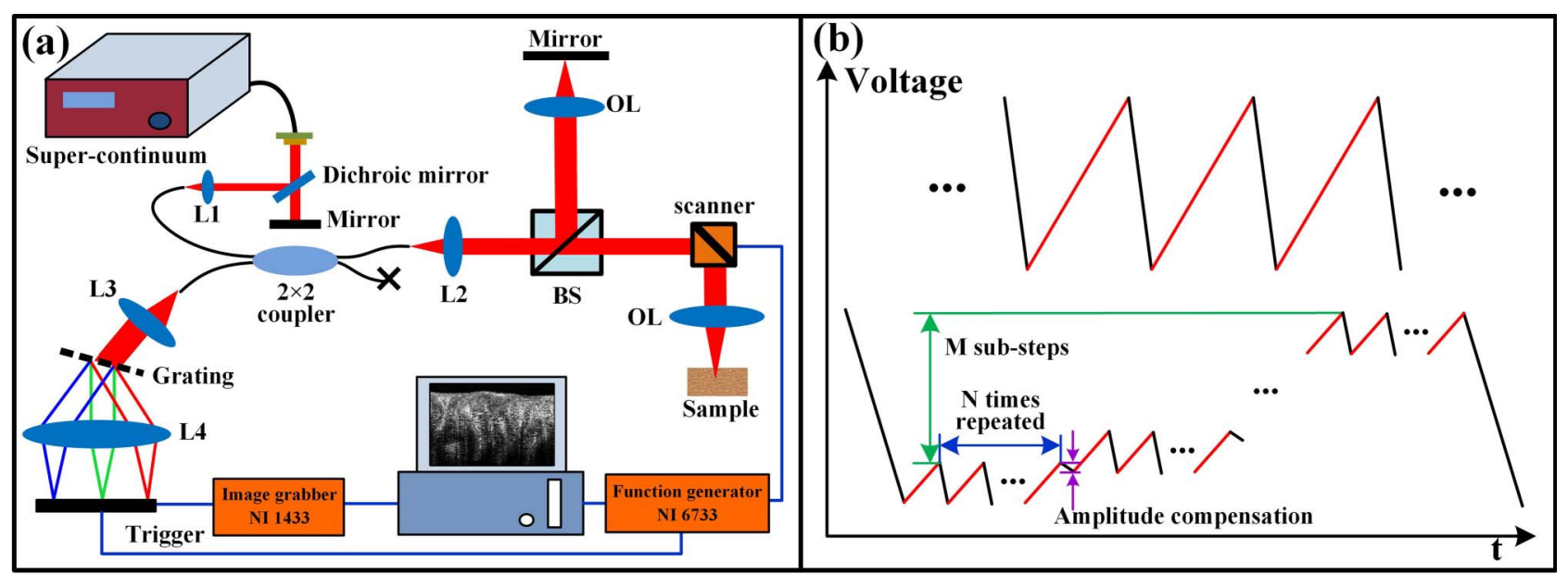

Fig. 1. (a) Illustration for the BD-MOCT system; L1-L4, achromatic lens; BS, beam splitter; OL, objective lens. (b) The illustration for the two scanning protocols. The top and the bottom panels are, respectively, the conventional raster scanning protocol and the B-scan-sectioned scanning protocol. The data acquisition occurs in forward scanning (red paths), and the duty cycle is 77\%. In the B-scan-sectioned scanning protocol, each full B-scan is divided into M sub-B-scans, and each sub-B-scan is repeated $N$ times. 
respectively. The corresponding sampling frequencies (Fs) for recording the dynamic signals were about $38 \mathrm{~Hz}$ and $286 \mathrm{~Hz}$ in the two scanning protocols. The data acquisition time for each dataset was $\sim 7.5 \mathrm{~s}$. The BD-MOCT system was located on a non-floated optical table to mimic clinical environmental applications.

To improve the precision of image stitching, we performed a calibration step to find the optimized compensation amplitude value [marked in Fig. 1(b)] between two adjacent sub-B-scans. Similar to our previous work ${ }^{[8]}$, we scanned a slightly tilted mirror to test the performance, and, meanwhile, the compensation value was slowly adjusted until a perfect image mosaic was achieved. For the BD-MOCT technique, each acquired dataset consisted of $4096 \times 136 \times 200 \times 8$ pixels. The first data processing step was to take out the extra four A-scans on each side of the sub-B-scans, and then we re-aligned the dataset into $4096 \times$ $1024 \times 200$ pixels based on the scanning sequences. Second, we performed FFT on each A-scan to calculate the structural images. After that, FFT was performed on the obtained structural dataset in the time direction for calculating the power spectrum at each pixel on the cross-sectional image. Finally, the pixel values within the three frequency ranges of $0-\mathrm{Fs} / 200$, Fs/ 200-Fs/40, and Fs/40-Fs/2 were averaged for generating the blue-, green-, and red-channel images of the output dynamic contrast images. The reason for applying the sampling frequency related three spectral bandwidths (instead of fixed spectral bandwidths) for comparison is to match the signal-to-noise ratios (SNRs) of the three channel images (between the two scanning protocols) by averaging the same number of pixels. The data process was performed with MATLAB (R2021a, MathWorks) on a work station (Intel Core i7, 16 GB memory), and the data processing time for the datasets of the conventional D-MOCT and BD-MOCT is $\sim 6.3 \mathrm{~s}$ and $\sim 6.5 \mathrm{~s}$, respectively.

\section{Results}

We first demonstrated the performance of BD-MOCT on onion core tissue imaging. The cells of onion tissue are big, and less scattering occurs inside of the cells due to the vacuoles. In this way, most scattered photons are from the cell walls, which make it easier to see the influence of bulk motions on the dynamic signal extraction procedure from the obtained dynamic contrast images. A small piece of tissue was extracted from the onion core and immediately immersed in a drop of saline on a glass slide for scanning. The obtained images are shown in Fig. 2. In comparing Figs. 2(a)-2(c) and Figs. 2(f)-2(h), we can see that the redand blue-channel images between the two methods are very close; but for the green-channel image in Fig. 2(b), the cell walls (especially the regions marked by the dashed yellow ellipse and yellow arrows) are much more blurred and brighter compared to Fig. 2(g). In comparison of the merged color images in Figs. 2(e) and $2(\mathrm{j})$, it can be seen that Fig. 2(j) has a more detailed colorful contrast for the dynamic signals, while almost all the structures are in white in Fig. 2(e) (even for the surface of the tissue). We also calculated the cross-correlation coefficients between the
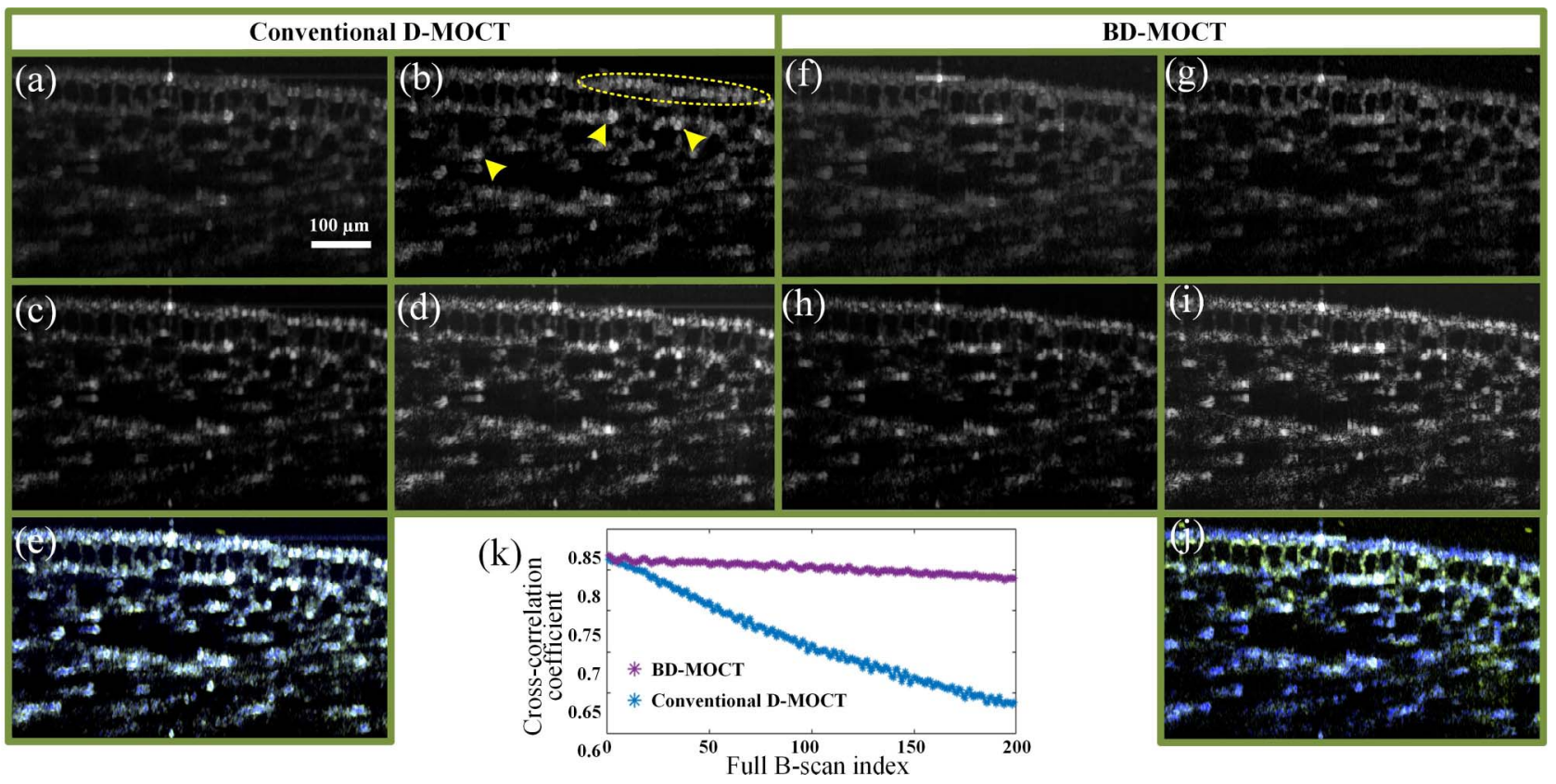

Fig. 2. Images of onion core tissue. (a)-(c) The red-, green-, and blue-channel images of the onion core tissue obtained by conventional D-MOCT. (d) The averaged structural image of the $200 \mathrm{~B}$-scans. (e) The merged dynamic contrast image. (f)-(h) The red-, green-, and blue-channel images of the onion core tissue obtained by BD-MOCT. (i) The averaged structural image of the 200 B-scans. (j) The merged dynamic contrast image. (k) The cross-correlation coefficients between the first B-scan and the following 199 B-scans of the two methods. Blue-channel image: 0-Fs/200; green-channel image: Fs/200-Fs/40; red-channel image: $\mathrm{Fs} / 40-\mathrm{Fs} / 2$. (a)-(j) share the same scale bar 
first B-scan and the following 199 B-scans for a quantitative comparison, and the plots are shown in Fig. 2(k). It is seen that the correlation degree of $\mathrm{BD}-\mathrm{MOCT}$ reduced much more gently than that of the conventional D-MOCT, demonstrating that bulk motions were suppressed well by the B-scan-sectioned scanning protocol.

An ex vivo experiment was further performed for testing the performance of $\mathrm{BD}-\mathrm{MOCT}$. In this experiment, we scanned a zebrafish's intestines with two scanning protocols and calculated both dynamic contrast images for comparison. The intestines were immediately immersed in cell culture medium after extraction for scanning. This study was approved by the Animal Research Ethics Committee at the university. The obtained results are shown in Fig. 3. In comparison of the three channel images of the two methods, we can see that Figs. 3(a) and 3(c) are very close to Figs. 3(f) and 3(h), respectively, which agrees well with the onion's results. However, the green-channel images [Figs. 3(b) and 3(g)] are apparently different, where Fig. 3(g) has richer dynamic information. The averaged structural images of Figs. 3(d) and 3(i) are also similar to each other. Comparing Figs. 3(e) and 3(j), we could see that Fig. 3(e) is globally redder, indicating that more dynamic signals were detected in the highfrequency band. Because the two images are from the same location of tissue sample, we believe the extra detected dynamic information in Fig. 3(e) was from bulk motions. From the two magnified local areas of Region I and Region II, we can see that some locations in Fig. 3(e) (such as those marked by yellow arrows) are blurred, and less sub-cellular information is achieved compared to Fig. 3(j). The results demonstrate that the proposed technique could effectively suppress the bulk motions and improve the quality of dynamic contrast images. Furthermore, we also calculated the SNR of the three channel images with the two local regions marked by the green and red rectangles in Fig. 3(d) for quantitative comparisons; the SNRs of Figs. 3(a)-3(c) are 31.9, 28.2, and 31.4 dB, respectively, while those of Figs. 3(f)-3(h) are 33.1, 27.4, and 30.2 dB, respectively. It could be seen that the B-scan-sectioned scanning protocol barely affected the image quality of each channel. The SNR was calculated by

$$
\mathrm{SNR}=20 \cdot \log \left(\bar{I}_{\mathrm{sig}} / \sigma_{\mathrm{bkg}}\right)
$$

where $\bar{I}_{\text {sig }}$ is the mean value of the local region marked by the red rectangle, and $\sigma_{\mathrm{bkg}}$ is the standard deviation of the local region marked by the green rectangle.

Since the BD-MOCT technique reduced the time interval between the consecutive stitched B-scans, the sampling frequency for recording the dynamic signals was improved, achieving a wider power spectrum for constructing D-MOCT images. We calculated the extra bandwidth signals (13.5-104.5 Hz) achieved by BD-MOCT over the conventional D-MOCT, and the result is shown in Fig. 4(a). Furthermore, the correlation coefficients between the first B-scan and the following 199 B-scans were also calculated for a quantitative comparison, as shown in Fig. 4(b). It is seen that the correlation degree of BDMOCT drops much more slowly than that of conventional DMOCT, which also agrees with the onion tissue's results. Because both moving scatterers and bulk motions could cause correlation degree loss, and the two datasets are from the same location of the tissue sample, we believe that the extra loss of blue

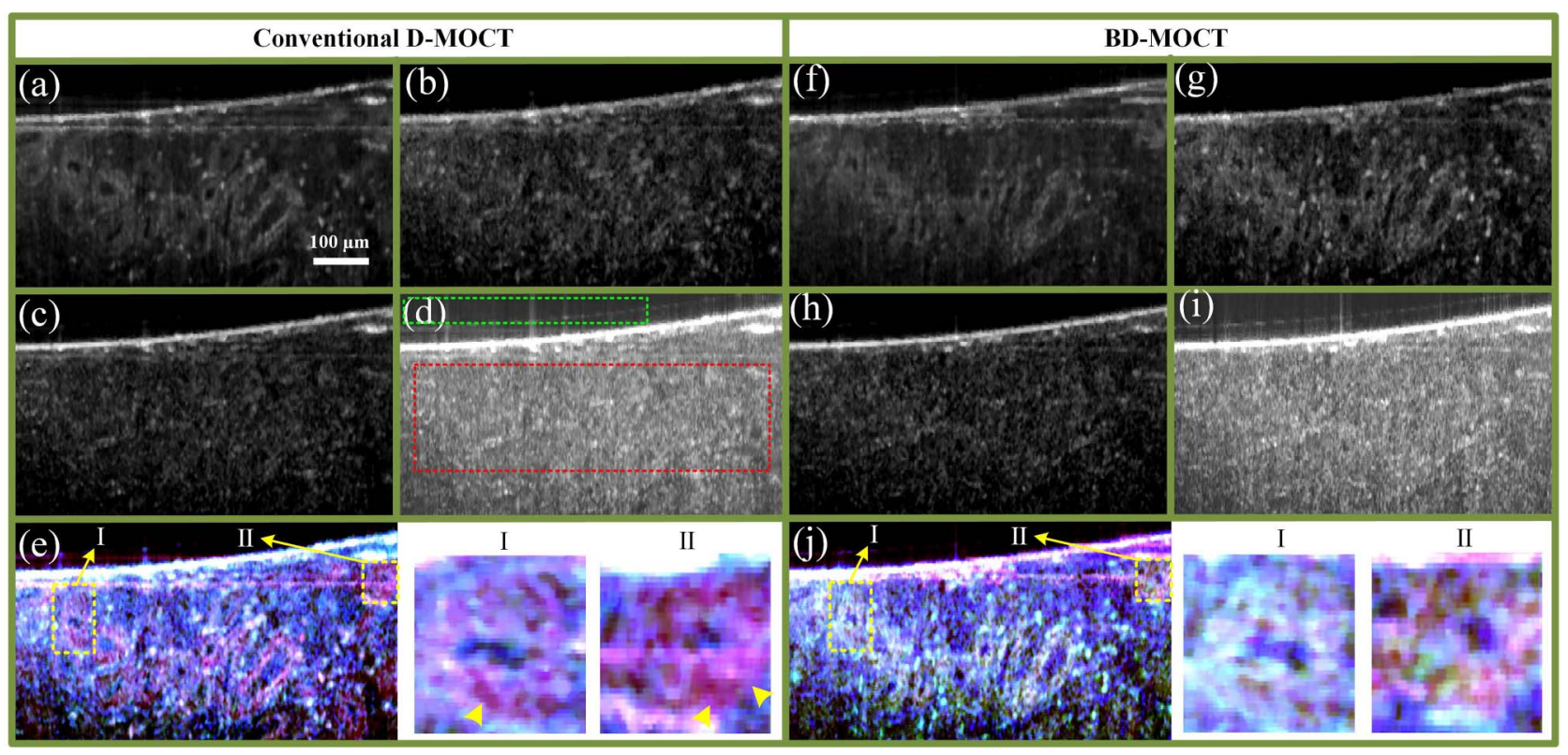

Fig. 3. Images of a zebrafish's intestines. (a)-(c) The red-, green-, and blue-channel images of a zebrafish's intestines obtained by conventional D-MOCT. (d) The averaged structural image of the 200 B-scans. (e) The merged dynamic contrast image. (f)-(h) The red-, green-, and blue-channel images of a zebrafish's intestines obtained by BD-MOCT. (i) The averaged structural image of the $200 \mathrm{~B}$-scans. (j) The merged dynamic contrast image. Blue-channel image: 0-Fs/200; green-channel image: Fs/200-Fs/40; red-channel image: Fs/40-Fs/2. (a)-(j) share the same scale bar. 

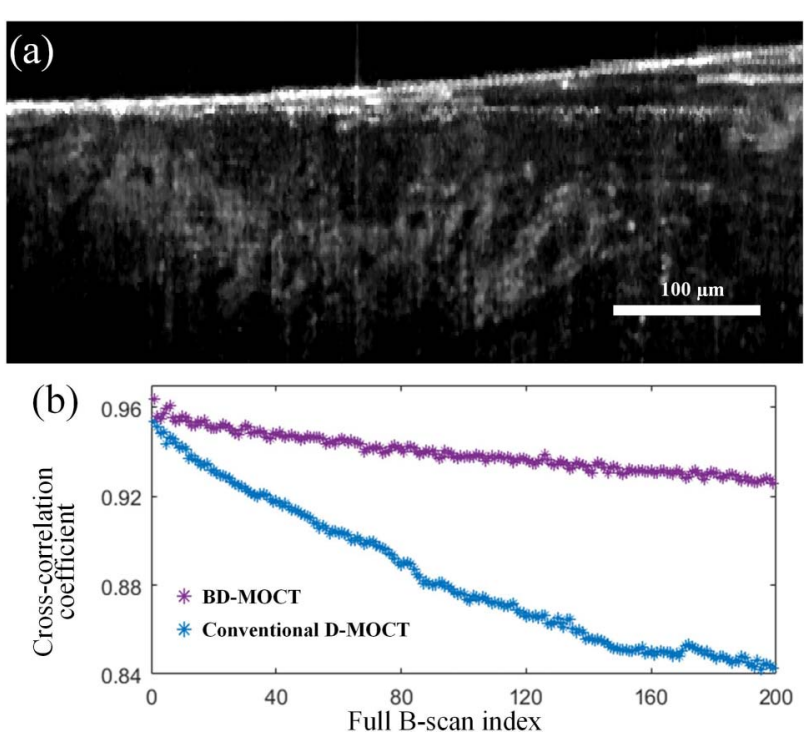

Fig. 4. (a) Image of the extra bandwidth (13.5-104.5 Hz) achieved by BD-MOCT over the conventional D-MOCT. (b) Cross-correlation coefficients between the first B-scan and the following 199 B-scans of the two methods.

plot compared to purple plot is caused by bulk motions, which demonstrates the performance of BD-MOCT.

\section{Discussion and Conclusion}

The D-MOCT technique achieves sub-cellular dynamic contrast by recording and analyzing the power spectrum of the backscattered signals (in time direction) at each spatial location. Because cell size is tiny and hundreds of B-scans need to be acquired for power spectrum calculation, bulk motions may severely degrade the quality of the obtained dynamic contrast images. An obvious way to suppress the bulk motions is to improve the A-scan rate $^{[9]}$, so the data acquisition could be completed during a small time period. However, MOCT is commonly based on the SDOCT scheme, which takes the advantage of ultrawide spectrum light sources (such as super-continuum light source and Ti:sapphire lasers), while the A-scan rate of SDOCT is limited by the line-scan camera, which is very challenging and expensive to be greatly improved. The B-scan-sectioned scanning protocol performed in this study modulates the sequences of fast scanning and repeat scanning for reducing the time interval between two consecutive B-scans. In this way, the bulk motion could be effectively suppressed, and it also achieves an improved sampling frequency for recording the dynamic signals, resulting in a wider bandwidth for storing extra dynamic information. Compared to the short-time Fourier-transform-based bulkmotion suppressing method (performed in Leung's work ${ }^{[6]}$ ), the post data processing algorithm of the proposed technique is easier. Compared to Münter's work ${ }^{[7]}$, in which only the axial sample shifts were compensated, the proposed technique could suppress both axial and lateral bulk motions.

In this work, we empirically scanned each location 200 times for sub-cellular dynamic information extraction based on
Leung' ${ }^{[6]}$ and Münter's ${ }^{[7]}$ studies. Higher image quality could be obviously achieved with more repeated B-scans, but the data acquisition time, data size, and data processing time would increase. Therefore, taking into consideration the tradeoff between the image quality and imaging speed, the repeat scanning number should be set based on the specific application. For example, if real-time screening is needed, the repeat scanning number needs to be reasonably reduced to decrease the time for data acquisition and data processing.

We divided each full B-scan into eight sub-B-scans to suppress bulk motions in this work, and, theoretically, this number could be higher to further reduce the time interval between them. However, the scanning efficiency (effective A-scans/total A-scans) drops as the sub-B-scan number increases, because eight extra A-scans were acquired for compensating the blurred regions caused by inertia of the galvo-mirror. The investigation on optimizing this time interval might be helpful for the ultrahigh-speed OCT systems, such as the $600 \mathrm{kHz}$ D-MOCT system $^{[9]}$.

Since D-MOCT is very sensitive to bulk motions because of high spatial resolutions, the proposed technique could effectively suppress the relatively weak bulk motions caused by tissue elasticity or environmental vibrations. For the strong bulk motions such as those that occurred during in vivo scanning, a few improvements on our current system might be needed, such as hardware speed and hand-held probe.

In summary, we present a BD-MOCT for achieving highquality sub-cellular dynamic contrast images, where each full $\mathrm{B}$-scan is divided into multiple sub-B-scans, and hundreds of repeated scans are performed before the sample beam moves to the next sub-B-scan. In this way, the time interval between the consecutive B-scans could be reduced multiple times to effectively suppress bulk motions. The clinical OCT systems are commonly placed on mobile carts (instead of floated optical tables); the environmental vibrations are inevitable and may severely degrade the quality of dynamic contrast images. The proposed technique may provide a way to overcome this kind of issue in clinical applications.

\section{Acknowledgement}

This work was supported by the Natural Science Foundation of Jiangsu Province (No. BK20210227).

\section{References}

1. C. Apelian, F. Harms, O. Thouvenin, and A. C. Boccara, "Dynamic full field optical coherence tomography: subcellular metabolic contrast revealed in tissues by interferometric signals temporal analysis," Biomed. Opt. Express 7, 1511 (2016).

2. J. Scholler, O. Thouvenin, K. Grieve, K. Groux, C. Boccara, V. Mazlin, M. Fink, J.-A. Sahel, and P. Xiao, "Probing dynamic processes in the eye at multiple spatial and temporal scales with multimodal full field OCT," Biomed. Opt. Express 10, 731 (2019).

3. J. Scholler, K. Groux, O. Goureau, J. A. Sahel, M. Fink, S. Reichman, C. Boccara, and K. Grieve, "Dynamic full-field optical coherence tomography: 3D live-imaging of retinal organoids," Light Sci. Appl. 9, 140 (2020). 
4. I. A. El-Sadek, A. Miyazawa, L. T. Shen, S. Makita, S. Fukuda, T. Yamashita, Y. Oka, P. Mukherjee, S. Matsusaka, T. Oshika, H. Kano, and Y. Yasuno, "Optical coherence tomography-based tissue dynamics imaging for longitudinal and drug response evaluation of tumor spheroids," Biomed. Opt. Express 11, 6231 (2020).

5. L. Liu, J. A. Gardecki, S. K. Nadkarni, J. D. Toussaint, Y. Yagi, B. E. Bouma, and G. J. Tearney, "Imaging the subcellular structure of human coronary atherosclerosis using micro-optical coherence tomography," Nat. Med. 17, 1010 (2011).

6. H. M. Leung, M. L. Wang, H. Osman, E. Abouei, C. MacAulay, M. Follen, J. A. Gardecki, and G. J. Tearney, "Imaging intracellular motion with dynamic micro-optical coherence tomography," Biomed. Opt. Express 11, 2768 (2020).
7. M. Münter, M. vom Endt, M. Pieper, M. Casper, M. Ahrens, T. Kohlfaerber, R. Rahmanzadeh, P. König, G. Hüttmann, and H. Schulz-Hildebrandt, "Dynamic contrast in scanning microscopic OCT," Opt. Lett. 45, 4766 (2020).

8. C. Chen, W. Shi, R. Reyes, and V. X. D. Yang, "Buffer-averaging supercontinuum source based spectral domain optical coherence tomography for high speed imaging," Biomed. Opt. Express 9, 6529 (2018).

9. M. Münter, M. Pieper, T. Kohlfaerber, E. Bodenstorfer, M. Ahrens, C. Winter, R. Huber, P. König, G. Hüttmann, and H. Schulz-Hildebrandt, "Microscopic optical coherence tomography (mOCT) at $600 \mathrm{kHz}$ for $4 \mathrm{D}$ volumetric imaging and dynamic contrast," Biomed. Opt. Express 12, 6024 (2021). 